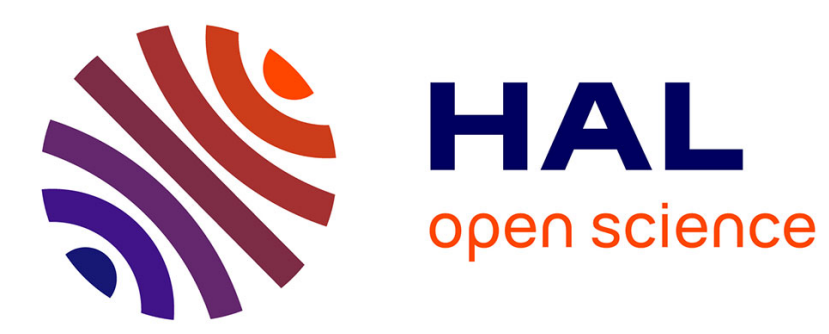

\title{
Ultrasonic determination of the particle concentration in model suspensions and ice slurry
}

\author{
Vincent Langlois, W Gautherin, J Laurent, L Royon, L Fournaison, A \\ Delahaye, X Jia
}

\section{- To cite this version:}

Vincent Langlois, W Gautherin, J Laurent, L Royon, L Fournaison, et al.. Ultrasonic determination of the particle concentration in model suspensions and ice slurry. International Journal of Refrigeration, 2011, 34 (8), pp.1972-1979. 10.1016/j.ijrefrig.2011.06.009 . hal-01158601

\section{HAL Id: hal-01158601 \\ https://hal.science/hal-01158601}

Submitted on 1 Jun 2015

HAL is a multi-disciplinary open access archive for the deposit and dissemination of scientific research documents, whether they are published or not. The documents may come from teaching and research institutions in France or abroad, or from public or private research centers.
L'archive ouverte pluridisciplinaire HAL, est destinée au dépôt et à la diffusion de documents scientifiques de niveau recherche, publiés ou non, émanant des établissements d'enseignement et de recherche français ou étrangers, des laboratoires publics ou privés. 


\title{
Ultrasonic determination of the particle concentration in model suspensions and ice slurry
}

\author{
V. Langlois ${ }^{1}$, W. Gautherin ${ }^{2}$, J. Laurent ${ }^{1}$, L. Royon $^{3,4}$, L. Fournaison ${ }^{2}$, A. Delahaye ${ }^{2}$, X. Jia ${ }^{1, *}$ \\ ${ }^{1}$ Université Paris Est, LPMDI, CNRS FRE 3300, 5 Bd Descartes, 77454 Marne-la-Vallée \\ cedex 2, France, \\ ${ }^{2}$ Unité de Recherche du Génie des Procédés Frigorifiques GPAN, Cemagref, 92163 Antony \\ cedex, France \\ ${ }^{3}$ Université Paris Diderot, Laboratoire MSC, CNRS UMR 7057, 75013 Paris, France \\ ${ }^{4}$ Université Paris Est, IUT de Marne la Vallée, 5, Bd Descartes, 77454 Marne-la-Vallée cedex \\ 2, France \\ * Corresponding author: Tel: +33 160957237; Fax: +33 160957297. E-mail: jia@univ-mlv.fr
}

\begin{abstract}
The development of ice slurry for refrigeration systems and the enhancement of its efficiency depend on an accurate control of the ice concentration. We present here an ultrasonic method capable to measure precisely the particle concentration in ice slurry. To calibrate the ultrasonic measurement, we first determine the sound velocity and attenuation in two model suspensions (glass beads/polyethylene glycol and polyethylene beads/vaseline oil) for different particle volume fractions. The experimental results show a good agreement with the predictions of the two-component models in the long-wavelength limit. Additionally, the sound attenuation reveals a clear signature of the aggregate formation in the nearly iso-dense suspension. We next conduct the measurement of the sound velocity in the polypropyleneglycol ice slurry where the ice concentration changes with temperature. The ice concentrations extracted from our sound velocity measurements are well consistent with the values determined from the binary phase diagram.
\end{abstract}

Keywords: Ultrasound, sound velocity, concentration, ice slurry. 


\section{Nomenclature}

$\begin{array}{lll}A_{n} & \text { sound amplitude in the medium } n & \text { [V] }\end{array}$

a particle radius [m]

$c_{n} \quad$ sound velocity in the medium $n \quad\left[\mathrm{~m} \mathrm{~s}^{-1}\right]$

$\begin{array}{lll}F_{L S} & \text { solid-liquid interaction force } & {[\mathrm{N}]}\end{array}$

$k$ wave number $\left[\mathrm{m}^{-1}\right]$

L distance of propagation [m]

$\begin{array}{lll}L_{A} & \text { aggregate size }\end{array}$

$\begin{array}{lll}P & \text { liquid pressure } & {[\mathrm{Pa}]}\end{array}$

$v_{j} \quad$ particle velocity of the constituent $j \quad\left[\mathrm{~m} \mathrm{~s}^{-1}\right]$

$X_{P P G} \quad$ mass fraction of PPG in the mixture suspending liquid [-]

$X_{i} \quad$ mass fraction of PPG in the initial suspending liquid

$\alpha_{n} \quad$ sound attenuation in the medium $n \quad\left[\mathrm{~m}^{-1}\right]$

$\begin{array}{lll}\delta & \text { viscous boundary layer thickness }\end{array}$

$\theta \quad$ temperature $\quad[\mathrm{K}]$

$\kappa_{j} \quad$ elastic modulus of the constituent $j \quad$ [Pa]

$\bar{\kappa} \quad$ effective elastic modulus of the suspension $\quad[\mathrm{Pa}]$

$\lambda \quad$ acoustic wavelength $\quad[\mathrm{m}]$

$\begin{array}{lll}\mu & \text { liquid viscosity } & {[\mathrm{Pa} \mathrm{s}]}\end{array}$

$\begin{array}{lll}\rho_{j} & \text { density of the constituent } j & {\left[\mathrm{~kg} \mathrm{~m}^{-3}\right]}\end{array}$

$\begin{array}{lll}\rho_{\text {eff }} & \text { effective density of the suspension } & {\left[\mathrm{kg} \mathrm{m}^{-3}\right]}\end{array}$

$\phi_{p} \quad$ particle volume fraction $\quad[-]$

$\phi_{p}^{W} \quad$ particle mass fraction

$\phi_{\text {ice }}$ mass fraction of ice particles [-]

$\phi_{\text {ice }}^{\text {Diag }}$ mass fraction of ice particles deduced from the binary diagram [-]

$\omega \quad$ angular frequency $(=2 \pi f)$ of sound wave $\quad\left[\mathrm{rad} \mathrm{s}^{-1}\right]$

Subscripts

$p \quad$ particle

$f \quad$ fluid

susp suspension 


\section{INTRODUCTION}

In refrigeration and air conditioning industry, the development of solid-liquid phasechange material slurry, as ice slurry is a great challenge concerning environment and energetic constrains. Indeed, it reduces drastically the total amount of refrigerant in a system and also, the global efficiency is increased compared with monophasic fluids due to the high thermal energy per volume unit of the slurry [Bellas and Tassou, 2005; Davies, 2005; Guilpart et al, 2006; Kauffeld et al 2010].The development of these complex fluids faces some major problems especially the control of the ice particle concentration which is connected to the amount of energy available for cold transportation. For efficient operations, industry requirements for the control of particle concentration include direct on-line measurement without dilution, reliable equipment and ease of calibration and standardization. Currently, principal techniques developed for controlling the quantity are based on the measurement of physical properties of the mixture, as the measurement of the heat thermal capacity, the density or the electrical conductance, which gives access to the mass fraction with a calibration curve previously established. Among these methods, conductimetry and density systems are on-line apparatus but their accuracies decreases when the solid and liquid densities are close and when the volume fraction is more than $10 \%$. Calorimetric technique would provide the most accurate system but their adaptation on line remains difficult. Currently, none of these solutions allows an accurate determination of the concentration of particles on line.

In this work, we describe the ultrasonic method for determining the ice volume fraction in the slurry. It is based on the general understanding of sound propagation in dispersed media, such as colloids, emulsions and suspensions, within the framework of the effective medium theory [McClements et al, 1993; Holmes et al, 1993; Hoyos et al 1994; Hibberd et al, 1999]. In a two-component non Brownian suspension of solid particles dispersed in a Newtonian fluid, the sound velocity dispersion and attenuation result from the relative motion between the solid phase and fluid phase and depend strongly on the solid volume fraction and the frequency [Harker and Temple, 1988; Atkinson and Kytömaa, 1992; Kytömaa, 1995]. By the adequate inverse procedure and under certain circumstances, the particle fraction can be deduced from the acoustic properties of suspensions. Such ultrasonic characterization methods are non-intrusive and can be applied to undiluted and optically opaque slurries [McClements et al, 1993], which provide the possible on-line measurements for monitoring the particle concentration in the suspension like ice-slurry. 
We report here the ultrasonic measurements conducted in the ice slurry with the ice mass fraction ranging from $0 \%$ to $30 \%$. To verify the applicability of the acoustic method, we first investigate the sound propagation at isothermal conditions in two model suspensions, namely, glass beads/Polyethylene glycol (PEG) and Polyethylene (PE) beads/Vaseline oil. The latter corresponds to a nearly iso-dense suspension. Next, we describe the experiments made in the water-propylene glycol ice slurry placed in laminar flow inside a specifically developed apparatus, at different temperature.

\section{SOUND PROPAGATION IN A MODEL SUSPENSION}

\subsection{Two-component model}

In this section, we describe briefly the sound propagation through a suspension of monodisperse spheres in a Newtonian liquid. Particular attention is focused on the suspensions of nearly iso-density. Most of the theoretical models [Harker and Temple, 1988; Atkinson and Kytömaa, 1992] deals with the limit case of the long-wavelength propagation, $\lambda \gg a$, with $a$ the particle radius. The associated sound velocity and the attenuation depend on the materials properties of the two phase constituents, the solid particle concentrations and the solid-liquid interactions. The interaction force $F_{L S}$ includes two contributions: an unsteady drag force and a buoyancy force due to the instantaneous pressure $P$ gradients:

$$
F_{L S}=\left[A \phi_{p}\left(v_{p}-v_{f}\right)+B \frac{D\left(v_{p}-v_{f}\right)}{D t}\right]+\phi_{p} \frac{\partial P}{\partial x}
$$

Here the coefficients $A=\frac{9 \mu}{2 a^{2}}\left(D\left(\phi_{p}\right)+\frac{a}{\delta}\right)$ and $B=\rho_{f}\left(C\left(\phi_{p}\right)+\frac{9}{4} \frac{\delta}{a}\right)$ depend on the viscous boundary layer thickness $\delta=\sqrt{2 \mu / \rho_{f}} \omega$, the functional forms $D$ and $C$ and the particle volume fraction $\phi_{p}$ [Harker and Temple, 1988; Atkinson and Kytömaa, 1992; Kytömaa, 1995]. $\rho_{j}$, $v_{j}$ are the density and velocity of the constituents, respectively; the subscript $p$ and $f$ denotes the particle and the fluid.

Starting from a specified particle-fluid interaction Eq. (1), one can derive the expression for the complex wave number $\mathrm{k}$ of a sound wave propagating in the suspension $k^{2}=\omega^{2}\left(\rho_{\text {eff }} / \bar{\kappa}\right)$ [Harker and Temple, 1988; Atkinson and Kytömaa, 1992], where $\bar{\kappa}$ represents the effective elastic modulus of the two-component suspension (Reuss average) $1 / \bar{\kappa}=\phi_{p} / \kappa_{p}+\left(1-\phi_{p}\right) / \kappa_{f}$ and $\rho_{\text {eff }}$ the effective density:

$$
\rho_{\text {eff }}=\rho_{f}+\left(\rho_{p}-\rho_{f}\right) \phi_{p} \frac{M^{\prime}(\omega)+\rho_{f}\left(1-\phi_{p}\right)}{M^{\prime}(\omega)+\rho^{*}\left(1-\phi_{p}\right)}
$$


with $\rho^{*}=\left(1-\phi_{p}\right) \rho_{p}+\phi_{p} \rho_{f}$ and $M^{I}(\omega)=B-i \frac{A}{\omega}$ [Harker and Temple 1988]. The phase velocity is thus obtained by $c_{\text {susp }}=\frac{\omega}{\Re(k)}$ and the attenuation by $\alpha_{\text {susp }}=\Im(k)$ where $\Re()$ and $\Im\left(\right.$ ) denote the real and imaginary parts of the argument. The explicit expressions of $c_{\text {susp }}$ and $\alpha_{\text {susp }}$ can be obtained in the two asymptotic cases, leading to, i) the viscous regime at low frequency $(\mathrm{LF})(a \ll \delta)$,

$$
\begin{aligned}
& c_{\text {susp }}=\left(\frac{\bar{\kappa}}{\bar{\rho}}\right)^{\frac{1}{2}} \\
& \alpha_{\text {susp }}=\frac{a^{2} \omega^{2}}{9 \mu}\left(\frac{\bar{\rho}}{\bar{\kappa}}\right)^{\frac{1}{2}} \frac{\left(1-\phi_{p}\right)\left(\rho_{p} \rho_{f}-\rho^{*} \bar{\rho}\right)}{\bar{\rho} f\left(\phi_{p}\right)}
\end{aligned}
$$

and, ii) the inertial regime at high frequency (HF) $(a \gg \delta)$,

$$
\begin{aligned}
& c_{\text {susp }}=\left(\frac{\bar{\kappa}}{\bar{\rho}}\right)^{\frac{1}{2}}\left[\frac{c+\left(1-\phi_{p}\right)\left(\rho^{*} / \rho_{f}\right)}{c+\left(1-\phi_{p}\right)\left(\rho_{p} / \bar{\rho}\right)}\right]^{\frac{1}{2}} \\
& \alpha_{\text {susp }}=\frac{9}{4 a}\left(\frac{\mu \omega}{2 \bar{k}}\right)^{\frac{1}{2}} \frac{\left(1-\phi_{p}\right)\left(\rho_{p} \rho_{f}-\rho^{*} \bar{\rho}\right)}{\left[\bar{\rho} C+\rho_{p}\left(1-\phi_{p}\right)\right]^{\frac{1}{2}}\left[\rho_{f} C+\rho^{*}\left(1-\phi_{p}\right)\right]^{\frac{3}{2}}}
\end{aligned}
$$

with $\bar{\rho}=\phi_{p} \rho_{p}+\left(1-\phi_{p}\right) \rho_{f}$.

Let us now investigate the suspensions with close densities between different phases. Introducing the relative difference between the densities of the phases $\delta \rho^{r}=\left(\rho_{p}-\rho_{f}\right) / \rho_{f}$ and assuming $\delta \rho^{r} \ll 1$, we obtain $\frac{\rho_{\text {eff }}}{\rho_{f}}=1+\phi_{p} \delta \rho^{r}+o\left(\delta \rho^{\prime 2}\right)$. The derivation of the wave number $k$ allows us to obtain the sound velocity and attenuation at the limiting case $\delta \rho^{r} \ll 1$,

$$
\begin{aligned}
& c_{\text {susp }}=\left(1-\frac{1}{2} \phi_{p} \delta \rho^{\prime}\right) \sqrt{\bar{\kappa} / \rho_{f}}+o\left(\delta \rho^{\prime 2}\right) \\
& \alpha_{\text {susp }}=-\left[\frac{\phi_{p}}{2} \frac{\left(\rho_{f}\left(1-\phi_{p}\right)\right)^{2}}{\left(\rho_{f}\left(1-\phi_{p}\right)+B\right)^{2}+\left(\frac{A}{\omega}\right)^{2}} \sqrt{\frac{\rho_{f}}{\bar{\kappa}}} \cdot \frac{A}{\rho_{f}}\right] \delta \rho^{\prime 2}+o\left(\delta \rho^{\prime 3}\right)
\end{aligned}
$$

Notice that for a nearly iso-dense suspension, since the inertia-viscous dissipation is expected to vanish at $\delta \rho^{r} \ll 1$, the sound velocity appears to be more sensitive than the attenuation to the presence of solid particles. We will see below that the attenuation measurement may infer the other loss mechanism in the suspensions. If the bulk modulus and density of each constituent are known, it is possible to determine the particle volume fraction from the sound velocity and consequently the particle mass fraction:

$$
\phi_{p}=\frac{1-\left(\frac{c_{\text {susp }}}{c_{f}}\right)^{2}}{\delta \rho^{\prime}+\left(\frac{c_{\text {susp }}}{c_{f}}\right)^{2}\left(\frac{k_{f}}{k_{p}}-1\right)}+o\left(\delta \rho^{\prime 2}\right)
$$




$$
\phi_{p}^{w}=\frac{\rho_{p} \phi_{p}}{\left(\rho_{p}-\rho_{f}\right) \phi_{p}+\rho_{f}}
$$

For the ideal case of iso-density suspension $\delta \rho^{r}=0$, Eq. (5a) recovers the Wood's relation for the sound velocity; the sound propagation becomes independent of the unsteady drag force. Moreover, if the unsteady drag force is function of $v_{p}-v_{f}$ and of its temporal derivate, the linearized equations [Atkinson and Kytömaa, 1992] lead to vanishing relative velocity and drag force. Therefore, there is no distinction between the viscous and inertial regimes in such a suspension.

\subsection{Experimental procedure}

We first conduct ultrasonic measurements on the model suspensions in isothermal conditions at ambient temperature (293 K). Two suspensions are investigated: one consists of the binary mixture of glass microbeads dispersed in polyethylene glycol (PEG300), the other of polyethylene microbeads in the nearly iso-dense vaseline oil. Table 1 gives the characteristics of the liquids and particles used in this work. These mixtures are contained in a cell $\left(4 \times 4 \times 20 \mathrm{~cm}^{3}\right)$ with the mass fraction of particles ranging from 5 to $40 \%$ and put in vacuum for a few minutes in order to remove the possible bubbles. The suspension is mixed by shaking gently up and down a plate and then allowed to settle. During this time the acoustic measurements are performed.

The settling phenomenon is observed for sufficient waiting time with the development of the concentration profile and the sedimentation front. In the latter, a boundary appears between the suspension and the liquid. In the suspensions studied, the Reynolds number is small leading to negligible inertial effects; while the Péclet number is large resulting in negligible Brownian motions [Hoyos et al, 1994; Segrè et al, 1997; Guazzelli, 2001]. After the settling is completed, the sediment attains a solid volume fraction of $56 \%$ for the glass/PEG mixture but only $43 \%$ for the PE/Vaseline mixture, being less than the volume fraction of a random loose packing. This observation could be understood by the fact that the critical surface tension of particles is very close to the surface tension of the liquid in the PE/Vaseline mixture, resulting in the aggregate formation (see below).

A broadband ultrasonic transducer of diameter $13 \mathrm{~mm}$ is used here as a source, centered at $1 \mathrm{MHz}$ with $-6 \mathrm{~dB}$ bandwidth from 0.7 to $1.3 \mathrm{MHz}$. It is mounted on the wall at the half-height of the cell and thus in direct contact with the suspension. Ten-cycle tone burst excitations at different frequencies are applied to the longitudinal source transducer. The 
pulsed ultrasound transmission is detected by another transducer placed on the opposite side. A digital oscilloscope is used to record the pulsed signals for further processing. Measurements performed in suspension are compared to those performed in the suspending liquid. Sound velocity and attenuation are determined from the time-of-flight and amplitude of the fifth maxima. We present below some experimental results in terms of relative sound velocity $\left(c_{\text {susp }}-c_{f}\right) / c_{f}$ and relative amplitude $A_{\text {susp }} / A_{f}$. The attenuation could be determined by the relative amplitude: $\alpha_{\text {susp }}=\ln \left(A_{\text {susp }} / A_{f}\right) / L$ with $L$ the distance of propagation $(L=40$ $\mathrm{mm})$.

\subsection{Results and discussions}

Fig. 1a shows the typical evolution of the sound velocity $c_{\text {susp }}$ and the amplitude transmission $A_{\text {susp }}$ during the sedimentation, measured at $f=700 \mathrm{kHz}$ in the glass/PEG suspension of $\phi_{p}=20 \%$ (volume fraction). For a given particle volume fraction, the measurements of ultrasound transmission remain quite stable for a duration where all our data are recorded, until the passage of the sedimentation front crossing the ultrasonic beam. We display, in Fig. 1b, the amplitude transmission measured as a function of frequency $(f)$ in the PE/Vaseline suspension of $\phi_{p}=10 \%$ at different instants after mixing. Significant attenuation is observed over the frequency range from 0.2 to $1.5 \mathrm{MHz}$. This finding is somewhat surprising for a nearly iso-dense suspension since the inertia-viscous dissipation would be vanishing, as expected by the above theory $(\$ 2.1)$. The scenario we propose is that the attenuation mechanism is likely due to the scattering loss when the acoustic wavelength $\lambda$ $\left(=c_{\text {susp }} / f\right)$ is comparable to the length scale of certain heterogeneity inside the suspension, such as the aggregate size $L_{A}(\gg 2 a)$ (see below, Fig. 4). At the earlier instant just after mixing or shearing the suspension, the observed attenuation varies with time especially at lower frequency $(f<600 \mathrm{kHz})$ or longer wavelength. This interesting result may be ascribed to the large-scale fluctuations of particles motions whose size $L_{L S}>L_{A}$ is closed to the cell size [Segrè et al, 1997]. Indeed, such a fluctuation decreases with time and reaches a stable ultimate size [Guazzelli, 2001].

Figure 2 depicts the relative sound velocity $\left(c_{\text {susp }}-c_{f}\right) / c_{f}$ and amplitude $A_{\text {susp }} / A_{f}$ measured as a function of particle volume fraction $\phi_{p}$ at different frequencies in the Glass/PEG suspension. The theoretical curves are also derived from the exact (Eq. 2) and 
asymptotic solutions (Eqs. 3 and 4) using the functional forms $D\left(\phi_{p}\right)=1 /\left(1-\phi_{\mathrm{p}}\right)^{2.65}$ [Kytömaa, 1995] and $\left.C\left(\phi_{p}\right)=\left(1-\phi_{p}\right) / 2\right)$ [Atkinson and Kytömaa, 1992]. The agreement between the theory and the experiment is fairly good. For a sound wave of $f=1 \mathrm{MHz}$ propagating in the PEG at $20^{\circ} \mathrm{C}$, the viscous boundary layer $\delta$ is $2.5 \mu \mathrm{m}$. Note however that in our experimental range, the asymptotic regimes, HF and LF, are not really satisfied.

We present, in Fig. 3a, the measured sound velocities versus frequency for different particle volume fraction $\phi_{p}$, which are consistent with the theoretical predictions. Fig. $3 \mathrm{~b}$ illustrates the amplitude transmission measured as a function of frequency for different $\phi_{p}$. These results show a significant attenuation beyond threshold frequency $f_{c}$ which depends on the particle volume fraction $\phi_{p}$. As mentioned above, the viscous dissipation is negligible in such nearly iso-dense suspension; the observed attenuation should be devoted to scattering loss associated with the aggregate formation [Haider et al, 2000]. Based on this picture, we may infer qualitatively the aggregates size from $L_{A} \sim \lambda=c_{\text {susp }} / f_{c}$. Fig. 4 displays the evolution of the deduced aggregate size as a function of the particle volume fraction. We find that more concentrated the suspension is, larger the aggregate size is.

In order to apply the acoustic method to characterize the suspension properties under various thermal circumstances (see below), we conduct finally the acoustic measurements in our model suspensions from $278 \mathrm{~K}$ to $308 \mathrm{~K}$. Figs. 5a and 5b show respectively the sound velocity and the amplitude transmission measured as a function of temperature in the Glass/PEG suspensions. The theoretical models accounting for the PEG viscosity variation versus temperature [Lee and Teja, 1990] agree quite well with the experimental results.

\section{MEASUREMENTS IN ICE SLURRY}

\subsection{Slurry preparation}

A small-scale loop circuit presented Fig. 6 is built for studying on-line ice slurry in laminar flow conditions. It consists of an ice slurry generator and a centrifugal pump. The centrifugal pump can deliver two flow rates depending on its setting. Tests were conducted with a $14 \mathrm{wt} \%$ polypropylene-glycol (PPG) ice slurry, performed with a brushed surface heat exchanger. The ice slurry is then circulated in the transparent ice slurry section. The mean diameter of the ice particles is around $60 \mu \mathrm{m}$ [Delahaye et al, 2010]. The measurement of the sound velocity is obtained with transducers mounted directly on the surface of the acrylic glass tube of $23 \mathrm{~mm}$ internal diameter. 
The protocol consists in slowly decreasing the temperature of the water polypropylene glycol mixture from $293 \mathrm{~K}$ towards $268 \mathrm{~K}$ at the rate of about $2 \mathrm{~K} / \mathrm{min}$. Indeed, the ice mass fraction in the slurry depends on the adjustment of the temperature in the generator. The sound velocity is determined by measuring the time-of-flight of transmitted pulses (Fig. 6), during all the process of slurry generation.

\subsection{Results and discussion}

Fig. 7 presents the sound velocity in the mixture as a function of temperature for two different laminar flow rates. Two regimes are clearly identified: the sound velocity $c_{\text {slurry }}$ decreases with lowering the temperature $\theta$ down to a threshold value $\theta_{i}=268.5 \mathrm{~K}$ whereas $c_{\text {slurry }}$ increases with further decreasing $\theta$ below $\theta_{i}$. This observation is well correlated with the phase transition in such a mixture, characterized by the binary diagram of the waterpolypropylene-glycol mixture (Fig 8). Indeed, from the initial conditions of the water/PPG mixture in the liquid state, namely, $\theta=\theta_{1}=293 \mathrm{~K}$ and $X_{P P G}=X_{i}=14 \%$ (with $X_{P P G}$ the mass fraction of PPG), the mixture undergoes a phase transition between the liquid state and the coexistence state of liquid-solid (i.e., ice slurry) at a temperature $\theta_{i}$ (depending on the initial mass fraction $X_{i}$ ). The mass fraction of ice particles $\phi_{i c e}$ formed at a temperature $\theta_{2}<\theta_{i}$ can be evaluated by the classical lever rule for a binary mixture in thermal equilibrium, $\phi_{\text {ice }}^{\text {Diag }}=\left(X_{P P G}\left(\theta_{2}\right)-X_{i}\right) / X_{P P G}\left(\theta_{2}\right)$ [Atkins and de Paula, 1986]. Here the mass fraction of the PPG in the suspending liquid, $X_{P P G}$, can be determined by the intersection of the horizontal line (crossing the temperature axis at $\theta_{2}$ ) with the liquidus curve, as shown in Fig. 8.

As shown in Fig. 9a, the variation of our measured sound velocity versus temperature in the liquid state for $\theta>\theta_{i}$ is in good agreement with the previous work [Zafarani - Moattar et al, 2004], conducted in the same kind of mixture for the higher temperature ranging from $283.15 \mathrm{~K}$ to $313.15 \mathrm{~K}$ (dashed curve). The slight discrepancy may be corrected by a modified density $\rho_{f}$ of the liquid mixture with a pre-factor (solid curve).

In order to extract the particle concentration in the ice slurry from the sound velocity (Eq. 6), we need to know the evolutions of the bulk modulus and density of both particles $\left(\kappa_{p}, \rho_{p}\right)$ and suspending liquid $\left(\kappa_{f}, \rho_{f}\right)$ as a function of temperature. For the ice particles, we can find the necessary information from the previous works [Marion and Jakubowski, 2004]. 
However, for the water-PPG mixture, the situation is more complicated since the associated acoustic properties depend not only on the temperature $\theta$ but also on the mass fraction of PPG in the suspending liquid $X_{P P G}(\theta)$ [Zafarani - Moattar et al, 2004]. This latter can be obtained from the binary phase diagram presented in Fig. 8. By a parabolic extrapolation, we may extend the evolutions of the bulk modulus and density of the aqueous PPG solution for the temperature range below $\theta_{i}$ from $267 \mathrm{~K}$ to $293 \mathrm{~K}$. We compare in Fig. $9 \mathrm{~b}$ the mass fraction of ice deduced from the sound velocity (Eq. 6) with that predicted by the binary phase diagram (Fig. 8). The agreement between the results obtained by the two methods is fairly good, confirming the applicability of our ultrasonic method for determining the particle concentration in ice slurries. Furthermore, it appears clear that considering the variation of the PPG mass fraction $X_{P P G}$ during the ice formation provides more consistent results than assuming a constant $X_{P P G}$.

\section{CONCLUSION}

In summary, we have verified the applicability of the long-wavelength sound propagation to determine the particle concentration in two model non-Brownian suspensions via the sound velocity. In the suspension of the PE beads dispersed in the nearly iso-dense Vaseline oil, little sound attenuation is detected due to the negligible inertia-viscous dissipation. However, the attenuation due to scattering is observed when the wavelength is comparable to the size of certain heterogeneity, e.g., associated with the aggregate formation. This latter affects slightly the sound velocity, but significantly the attenuation thus allowing us to infer the structural properties of suspensions.

On the other hand, our acoustic method is also applied to measure the particle concentration in ice slurry as a function of temperature. The mass fraction of the ice particles deduced from the measured sound velocity agrees well with the value obtained from the conventional binary phase diagram. This inverse method via ultrasonic measurements can be further improved if one accounts for more accurately the mass fraction change in binary aqueous solution during the ice crystal formation. Our results reveal the possibility to develop the adequate ultrasonic method for on-line measurement of the ice particle fraction and of the structure of ice slurry under different operating factors such as temperature and flow rate.

\section{Acknowledgments}


This study is supported by the Energy Program of the French CNRS. We thank P. Mills for the helpful discussions on the sedimentation, H. Sizun and R. Hunlede for the technical assistances on the experimental set-up.

\section{References:}

Atkins, P., de Paula, J. Physical Chemistry, Oxford University Press, Oxford, 1986, p. 182

Atkinson C.M., Kytömaa H.K., 1992. "Acoustic wave speed and attenuation in suspensions." Int. J. Multiphase Flow 18, 577-592

Bellas, I., Tassou, S.A., 2005. "Present and future applications of ice slurries.” Int. J. Refr. 28, $115-121$

Davies, T.W., 2005. "Slurry ice as a heat transfer fluid with a large number of application domains.” Int. J. Refr. 28, 108-114

Delahaye, A., Fournaison, L., Guilpart, J., 2010. "Characterisation of ice and THF hydrate slurry crystal size distribution by microscopic observation method.” Int. J. Refr. 33, 16391647

Guazzelli, E., 2001. "Evolution of particle-velocity correlations in sedimentation." Phys. Fluids 13, 1537-1540

Guilpart, J., Stamatiou, E., Delahaye, A., Fournaison, L., 2006 “ Comparison of the performance of different ice slurry types depending on the application temperature." Int. J. Refr. 29, 781-78

Haider L, Snabre P, Boynard M., 2000. "Rheo-acoustical study of the shear disruption of reversible aggregates. Ultrasound scattering from concentrated suspensions of red cell aggregates.” J. Acoust. Soc. Am. 107, 1715-1726

Harker, A.H., Temple, J.A.G., 1988. "Velocity and attenuation of ultrasound in suspensions of particles in fluids.” J. Phys. D: Appl. Phys. 21, 1576-1588

Hibberd, D.J., Robinson, B.H., Robins, M.M., 1999. "Ultrasonic characterisation of colloidal dispersions: detection of flocculation and adsorbed layers." Colloids and Surfaces B: Biointerfaces 12, 359-371

Holmes, K.A., Challis, E.R., Wedlock, D.J., 1993. “A wide bandwidth study of ultrasound velocity and attenuation in suspension: Comparison of theory with experimental measurement.” J. Colloid Interface Sci. 156, 261-268. 
Hoyos, M., Bacri, J.C., Martin, J., Salin, D., 1994. "A study of the sedimentation of noncolloidal bidisperse, concentrated suspensions by an acoustic technique." Phys. Fluids 6, 3809-3817

Kauffeld, M., Wang, M.J., Goldstein, V., Kasza, K.E., 2010. "Ice slurry applications.” Int. J. Refr. 33, 1491-1505

Kytömaa H.K., 1995. “Theory of sound propagation in suspensions: a guide to particle size and concentration characterization.” Powder Technol. 82, 115-121

Lee R.J., Teja, A.S., 1990. "Viscosities of Polyethylene glycols.” J. Chem. Eng. Data 35, 385387

Marion, G.M., Jakubowski, S.D., 2004. "The compressibility of ice to 2.0 kbar." Cold Regions Science and Technology 38, 211-218

McClements, D., Dickinson, Dungan, R.S., Kinsela, E.J., Povey, M.J.W., 1993. "Effect of emulsifier type on the crystallization of oil-in-water emulsions containing a mixture of solid and liquid droplets.” J. Colloid Interface Sci. 160, 279-293.

Segrè, P.N., Herbolzheimer, E., Chaikin, P.M., 1997. "Long-range correlations in sedimentation.” Phys. Rev. Lett. 79, 2574

Zafarani - Moattar, M.T., Samadi F. Sadeghi R., 2004. "Volumetric and ultrasonic studies of the system (water+polypropylene glycol 400) at temperatures from (283.15 to 313.15) K." J. Chem. Thermodynamics 36, 871-875 
Table 1 Characteristics of liquids and particles.

\begin{tabular}{lll}
\hline Liquid/Particle & PEG/glass & Vaseline oil/PE \\
\hline Liquid & PEG 300 & Vaseline oil \\
Density $\rho_{f}\left(\mathrm{~kg} / \mathrm{m}^{3}\right)$ & 1125 & 860 \\
Bulk modulus $\kappa_{f}(\mathrm{GPa})$ & 2.91 & 1.80 \\
Viscosity $\mu(\mathrm{Pa} . \mathrm{s})\left(20^{\circ} \mathrm{C}\right)$ & 0.1 & 0.215 \\
Surface tension $\gamma(\mathrm{mN} / \mathrm{m})\left(20^{\circ} \mathrm{C}\right)$ & $40-45$ & 32 \\
\hline Particle & Glass & Polyethylene \\
Density $\rho_{p}\left(\mathrm{~kg} / \mathrm{m}^{3}\right)$ & 2485 & 940 \\
Bulk modulus $\kappa_{p}(\mathrm{GPa})$ & 42.6 & 4.38 \\
Mean particle radius $a(\mu \mathrm{m})$ & 37.5 & 75 \\
Critical surface tension $\gamma_{c}(\mathrm{mN} / \mathrm{m})$ & $70-100$ & 34 \\
\hline
\end{tabular}




\section{Figure captions}

- Figure 1: (a) Glass/PEG suspension: the sound velocity difference $\left(c_{\text {susp }}-c_{f}\right) / c_{f}$ and the amplitude transmission $A_{\text {susp }} / A_{f}$, measured as a function of the waiting time (b) PE/Vaseline oil suspension: frequency profiles of $A_{\text {susp }} / A_{f}$ measured at the different waiting time.

- Figure 2: (a) Relative change of the sound velocity $\left(c_{s u s p}-c_{f}\right) / c_{f}$, (b) relative amplitude transmission $A_{\text {susp }} / A_{f}$ measured as a function of the volume solid fraction $\phi_{p}$ at different frequencies in Glass/PEG suspensions. Solid, dashed and dotted curves correspond to the theoretical predictions by Eqs. 2, 3 and 4.

- Figure 3: (a) Relative change of the sound velocity $\left(c_{s u s p}-c_{f}\right) / c_{f}$, and (b) relative amplitude transmission $A_{s u s p} / A_{f}$ measured as a function of frequency for different volume solid fractions $\phi_{p}$ in PE/Vaseline oil suspensions. Solid curves correspond to the theoretical predictions by Eqs. 2 and 5. Inset: attenuations predicted by Eq. $5 \mathrm{~b}$.

- Figure 4: Deduced aggregates size $L_{A}$ as a function of the particle volume fraction $\phi_{p}$.

- Figure 5: (a) Relative change of the sound velocity $\left(c_{s u s p}-c_{f}\right) / c_{f}$, and (b) relative amplitude transmission $A_{\text {susp }} / A_{f}$ measured as a function of temperature $\theta$ in glass/PEG suspensions. Solid and dashed lines correspond to the predictions by Eq. 2 with or without accounting for the liquid viscosity change with temperature, respectively.

- Figure 6: Experimental set-up for the ice slurry generation and flowing monitored by ultrasonic measurements.

- Figure 7: Sound velocity of ice slurry measured as a function of temperature during cooling for two flowing rate.

- Figure 8: binary phase diagram of the PPG/water mixture. The liquid state and the state of liquid + ice coexistence are separated by the liquidus curve.

- Figure 9: (a) Comparison between our experimental data (empty square in blue) and the results extrapolated from the [Zafarani - Moattar et al, 2004] (dashed curve) in the PPG/water mixture in the liquid state. For a better fit with our data (solid curve), the density $\rho_{f}$ is corrected by a pre-factor. (b) Comparison between the ice mass fraction deduced from sound velocity with $X_{P P G}$ variable (filled circles in red) or constant (empty circles in green) in the inverse procedure, and the ice mass fraction from the binary diagram $\phi_{\text {ice }}^{\text {Diag }}$ (solid curve). 


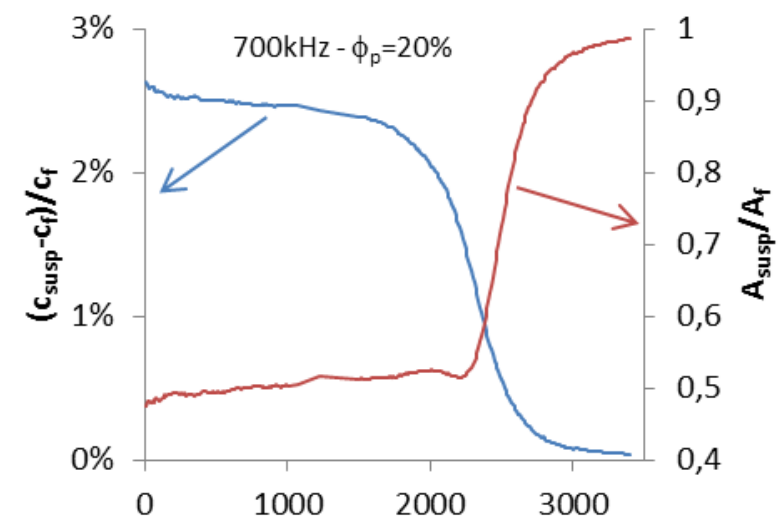

(a)

waiting time (s)

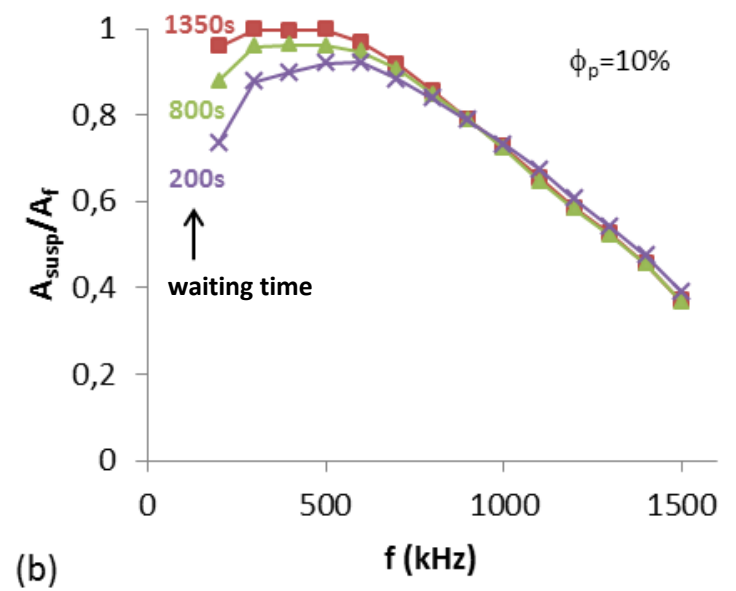

Figure 1 

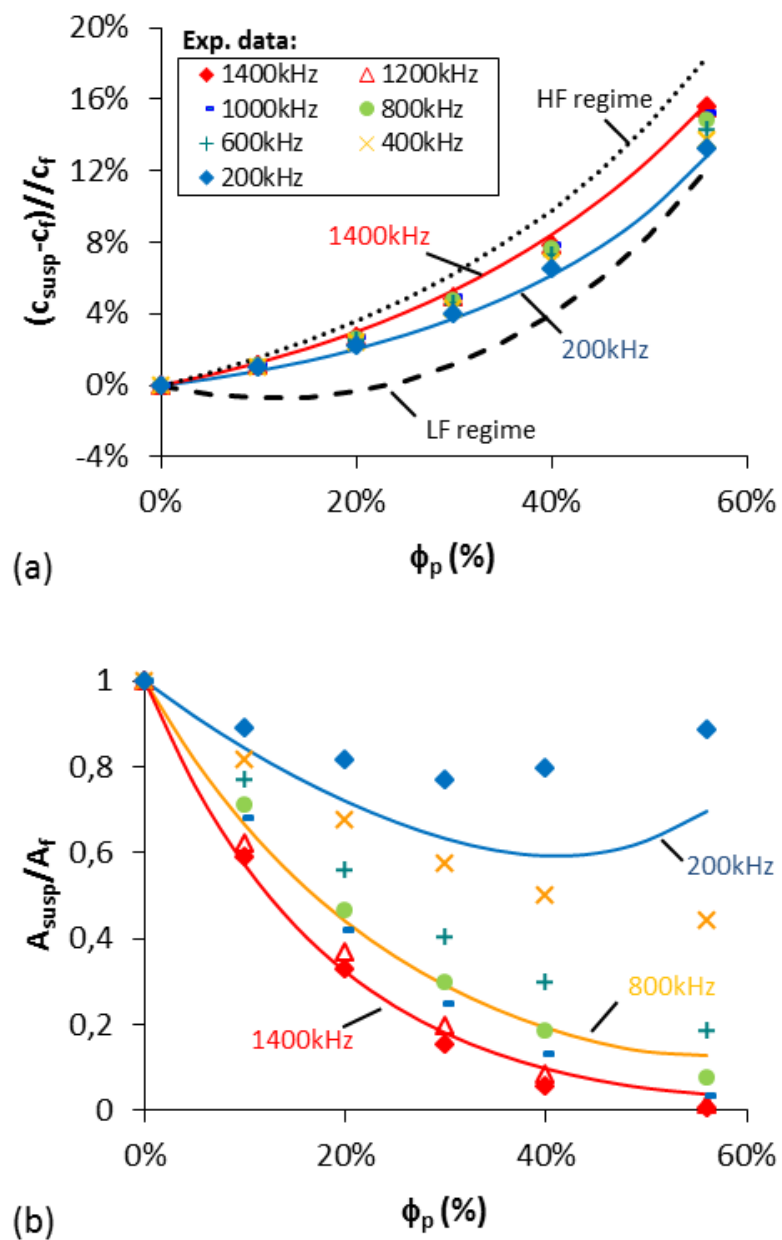

Figure 2 

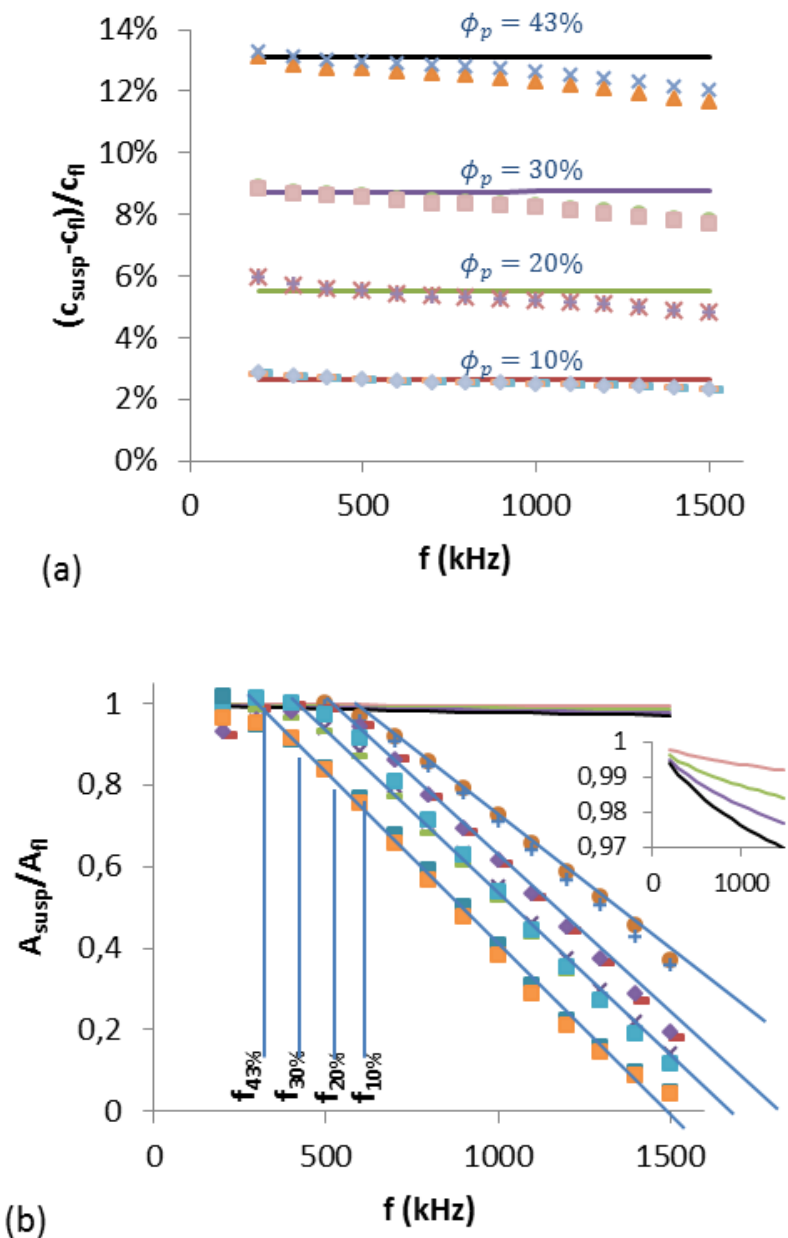

Figure 3 


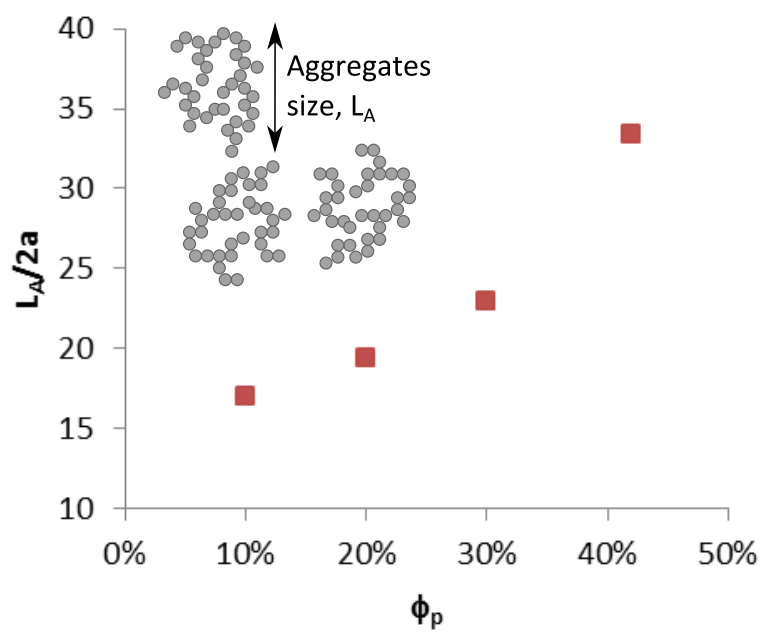

Figure 4 

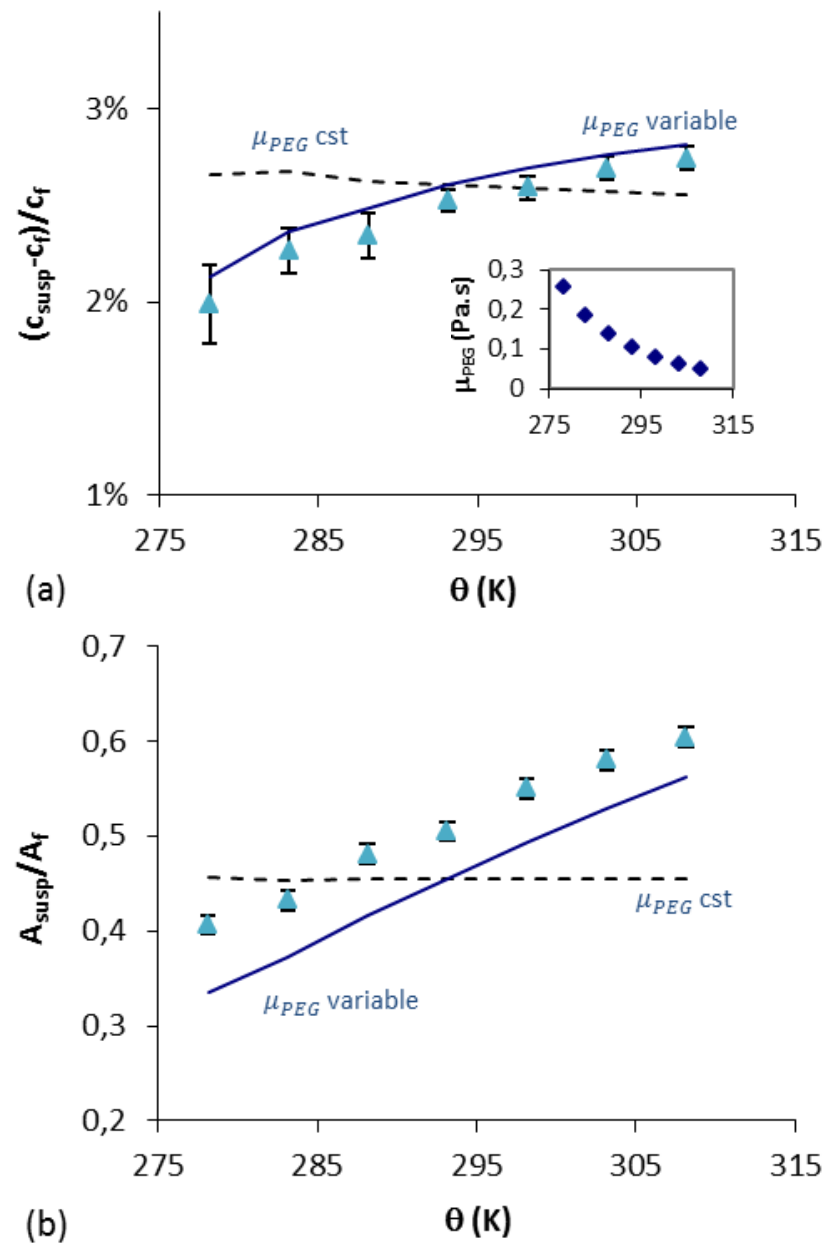

Figure 5 


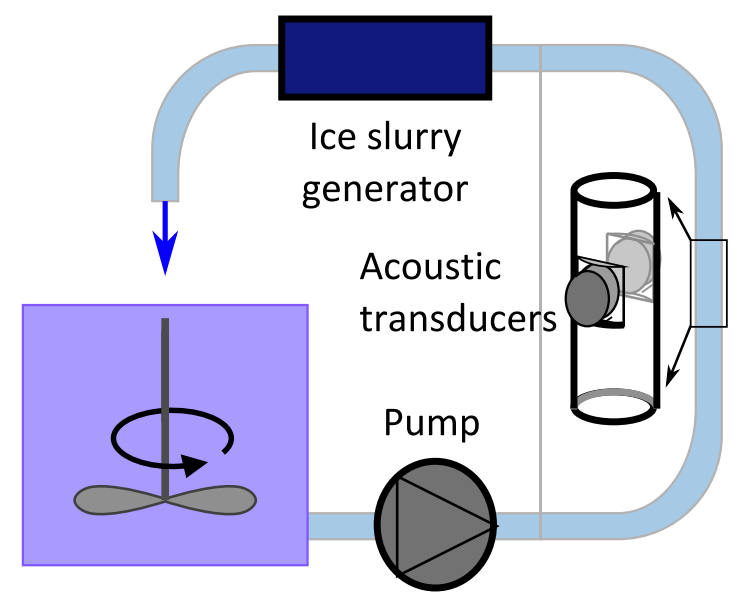

Figure 6 


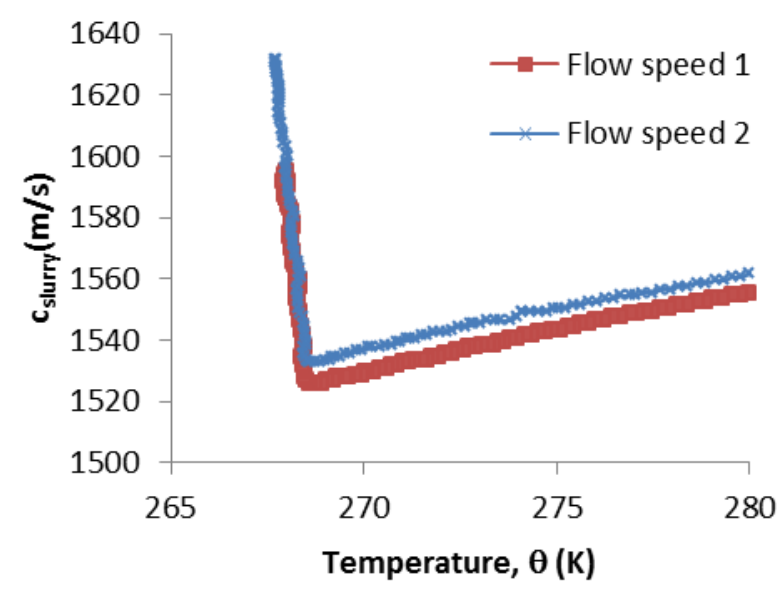

Figure 7 


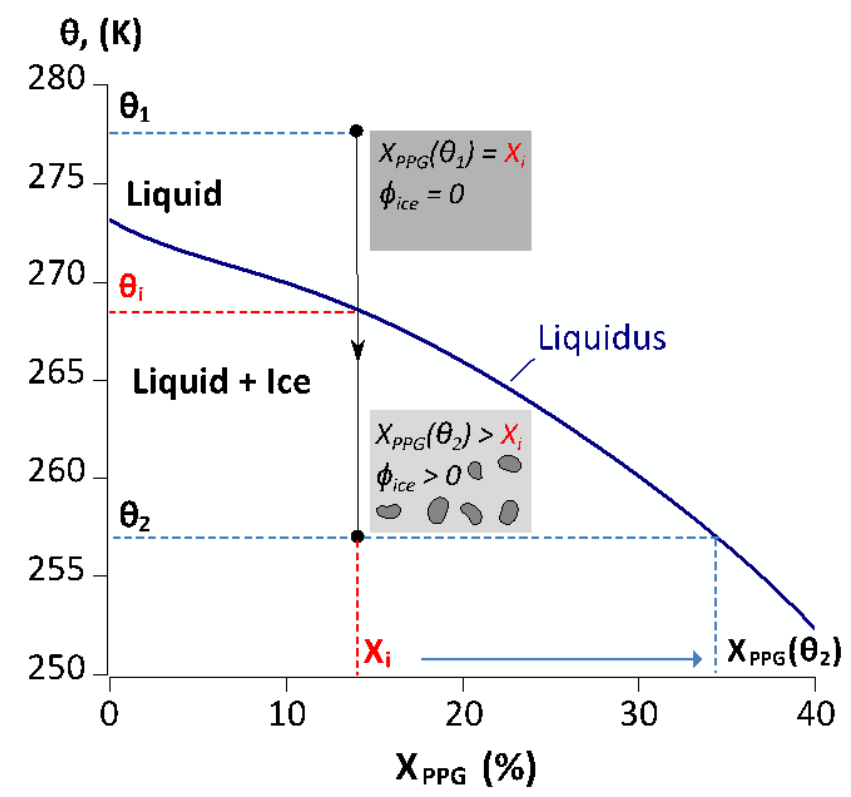

Figure 8 

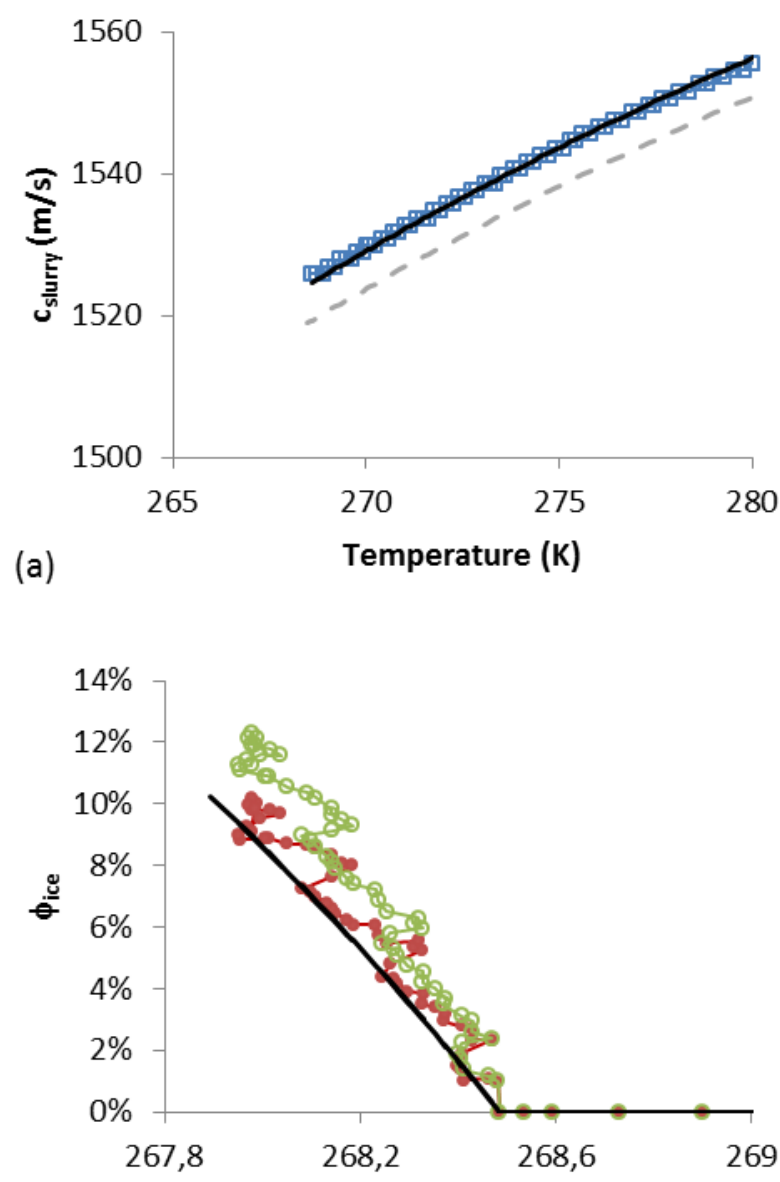

(b)

temperature (K)

Figure 9 